\title{
Editorial
}

\section{Nanostructural Biomaterials and Applications}

\author{
Mingdong Dong, ${ }^{1}$ Lei Liu, ${ }^{2}$ and Shuai Zhang $^{3}$ \\ ${ }^{1}$ Interdisciplinary Nanoscience Center (iNANO), Aarhus University, 8000 Aarhus, Denmark \\ ${ }^{2}$ Institute for Advanced Materials, Jiangsu University, Zhenjiang 212013, China \\ ${ }^{3}$ Physical Sciences Division, Pacific Northwest National Laboratory, Richland, WA 99352, USA
}

Correspondence should be addressed to Mingdong Dong; dong@inano.au.dk

Received 6 September 2016; Accepted 6 September 2016

Copyright (C) 2016 Mingdong Dong et al. This is an open access article distributed under the Creative Commons Attribution License, which permits unrestricted use, distribution, and reproduction in any medium, provided the original work is properly cited.

Nanostructure is the structure, at least one of whose dimensions is in nanoscale, normally between $0.1 \mathrm{~nm}$ and $100 \mathrm{~nm}$. With the recent exciting progress of nanoscience and nanotechnology, kinds of materials with nanostructures are being discovered and synthesized every day. As being one of the key subcatalogs, biomaterials with nanostructures are rapidly developing. The integration of nanostructures into biomaterials gives them unique structures and special and inspiring properties. Hence, a variety of nanostructural biomaterials have been applied in many aspects of daily life, like medical treatment and diagnosis. This field is still fast developing, and it has significant effects on improving our world. In this special issue, we collect three original works and two overviews from the researchers in Thailand, China, Denmark, and Germany. They present to people the promising applications of nanostructural biomaterials in tissue engineering, drug delivery, and disease diagnosis. These works aim to inspire further researches and efforts focusing on biofunctionalized nanomaterials and the corresponding applications.

S. Sirivisoot et al. in Thailand and X. Deng et al. in China have provided two marvelous cases in bone-tissue engineering with biological composite nanomaterials. S. Sirivisoot et al. (in "Improved Bonding Strength of Hydroxyapatite on Titanium Dioxide Nanotube Arrays following Alkaline Pretreatment for Orthopedic Implants") have focused on introducing nanostructures on Ti implant, in order to influence the further adsorbed hydroxyapatite (HA). They used alkaline to pretreat titanium dioxide nanotubes (ATi) on $\mathrm{Ti}$ surface, which is of capability to accelerate the formation of HA layer on top and tune the orientation. The synthesized
HA-ATi nanocomposite successfully mimics natural bone tissue and improves bioactivity of this type of orthopedic implants. That makes it a kind of new approach toward producing orthopedic implants with much longer functional life.

Unlike S. Sirivisoot el al.s focus, X. Deng et al. (in "Enhanced Stem Cell Osteogenic Differentiation by Bioactive Glass Functionalized Graphene Oxide Substrates") put their efforts to manipulate stem cell osteogenesis, in order to regenerate bone tissue. They utilized sol-gel method to synthesize graphene oxide (GO) and bioactive glasses (BG) hybrid scaffold. This new kind of biofunctional nanomaterial keeps the biological properties from the parents' materials. The following cellular activity analysis provided promising evidence to demonstrate that the hybrid scaffold, with unique nanostructure and biological properties, is of capability to trigger osteogenic differentiation of mesenchymal stem cells. That is a positive sign for the potential application of GO hybrid materials in bone restoration.

The hybrid nanomaterial could have medical applications other than the candidates for medical treatment. J. Liang et al. from China reported the usage of Au nanorod- (AuNR-) peptide hybrid for tumor diagnosis (in "Micro-CT Imaging of RGD-Conjugated Gold Nanorods Targeting Tumor In Vivo"). They conjugated AuNRs with peptide RGD, which is a kind of receptor targeting some tumor cells and tumor neovasculature overexpressed Integrin $\alpha_{v} \beta_{3}$. The conjugated nanoparticles eliminated the cytotoxicity of AuNRs, but they improve the ability to target tumor cells in vivo and in vitro. The micro-CT results also prove their feasibility to target tumor models. And the uptaken level of AuNRs-RGD 
nanoparticles is determined by the expression level of Integrin $\alpha_{v} \beta_{3}$ from tumor cells. All of the mentioned advantages give the authors a kind of promising candidate to diagnose tumors via micro-CT.

In other researches, hybrid nanoparticles have been used as nanocarriers to deliver drugs. T. Scheper et al. from Germany introduced and reviewed the application of noisome as nanodrug carriers. Noisome is self-associated by nonionic surfactants and cholesterol; and people have proved that it has several key advantages to deliver drugs, like biocompatibility, high stability in creature bodies, and feasibility to target specific site by controlled manner. The authors systematically introduced the structure and component of noisome. They further summarized the available methods to prepare and characterize noisome nanocarriers. In the end, the recent applications of noisome in drug delivery as nanocarriers were reviewed. We believe both academic researcher and therapeutic engineers will be inspired from this detailed review.

In nanoscience and nanotechnology, developing the techniques to characterize and evaluate nanostructures and nanomaterials is of the same importance as their fabrication and synthesis, and corresponding applications. M. Dong and his colleagues from Denmark, Germany, and China reviewed the applications of atomic force microscopy (AFM) and AFM based single-molecular force spectroscopy (AFMSMFS) to characterize inter- and intramolecular interactions of amyloid self-assembly nanofibril, in order to understand the mechanism of amyloidogenesis on nanoscale. Besides, they also introduced the idea to consider amyloid fibrils as nanomaterials, due to the outstanding mechanical properties and the corresponding potentials. This review will also inspire the readers from the relevant fields of AFM and AFM-SMFS to characterize and evaluate other kinds of biofunctionalized nanomaterials.

In summary, all these authors present the feasibility of nanostructural biomaterials in a number of aspects and several promising bioapplications. They convince people of the attractive future of biofunctional nanomaterials in daily life, especially in medical related fields. Hopefully, this special issue will inspire the ideas to synthesize new biofunctionalized nanomaterials and the corresponding applications. We wish that it also raises people's enthusiastic efforts in related researches.

Mingdong Dong

Lei Liu

Shuai Zhang 

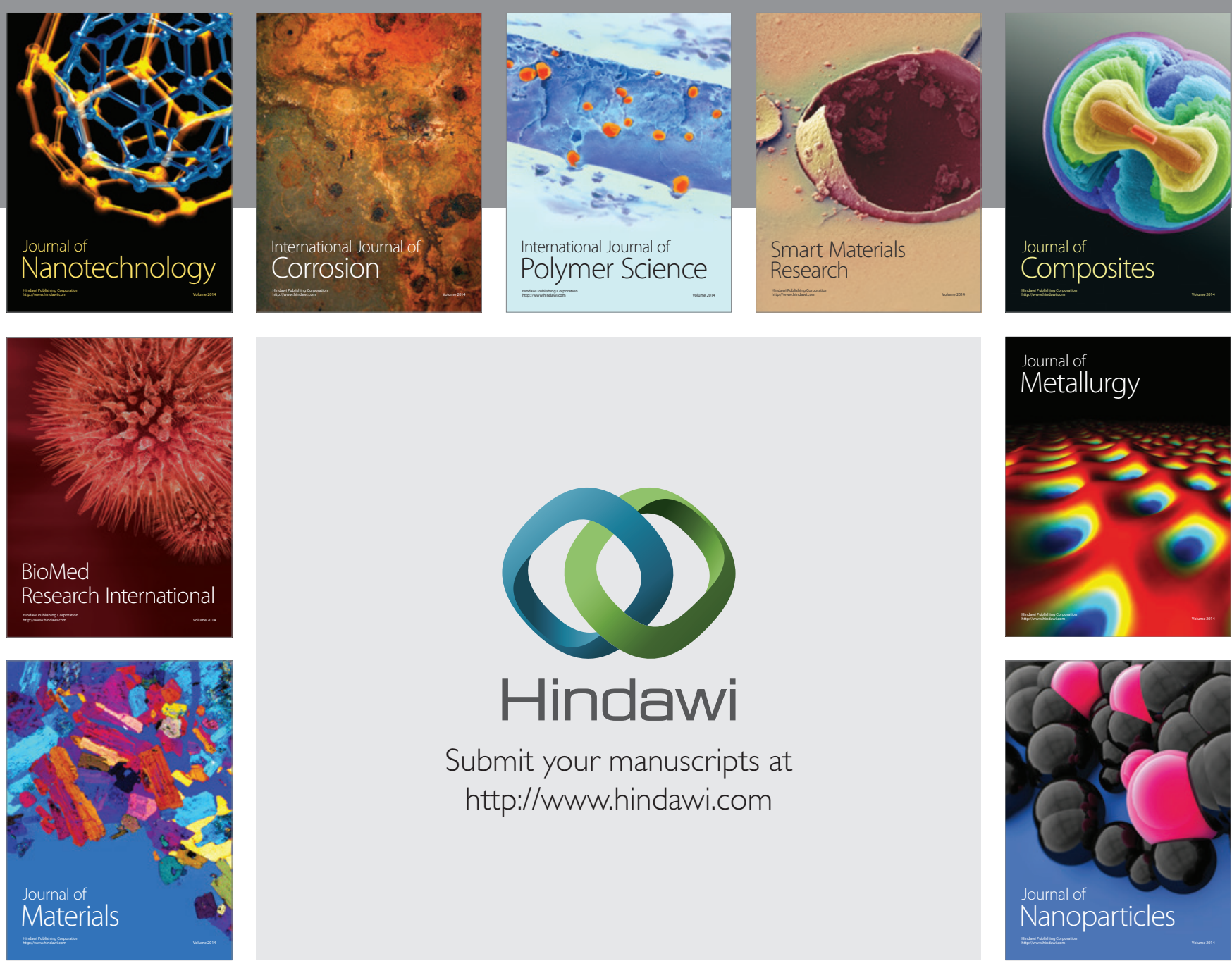

\section{Hindawi}

Submit your manuscripts at

http://www.hindawi.com

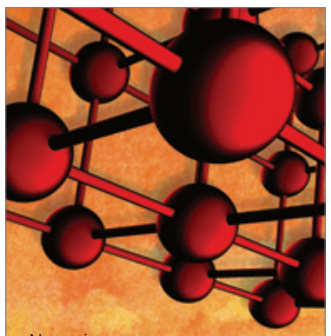

Materials Science and Engineering
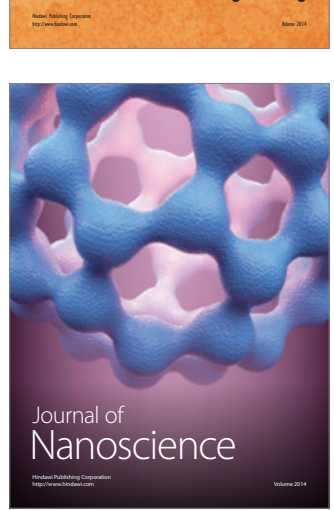
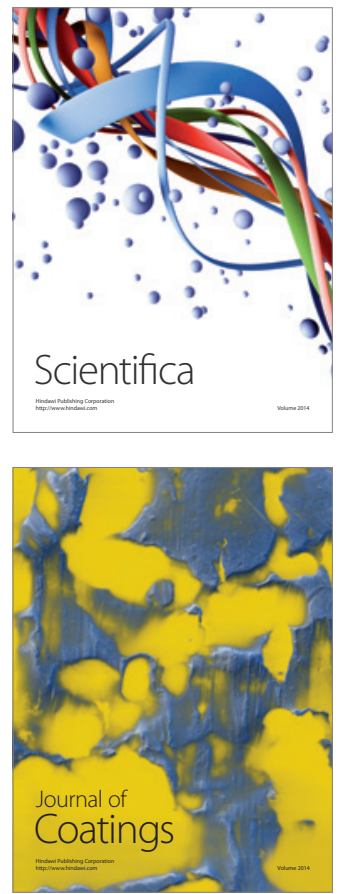
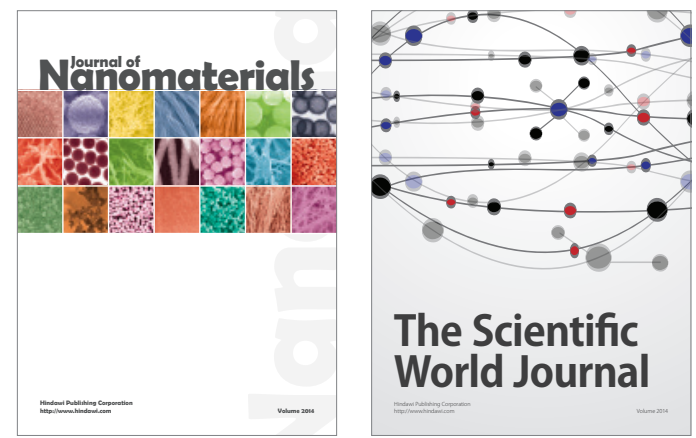

The Scientific World Journal
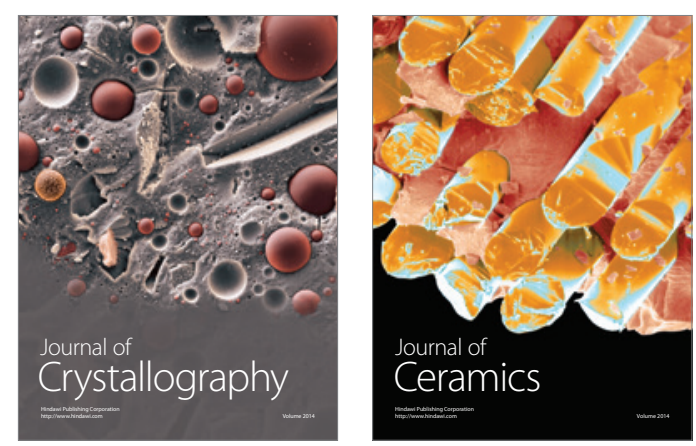
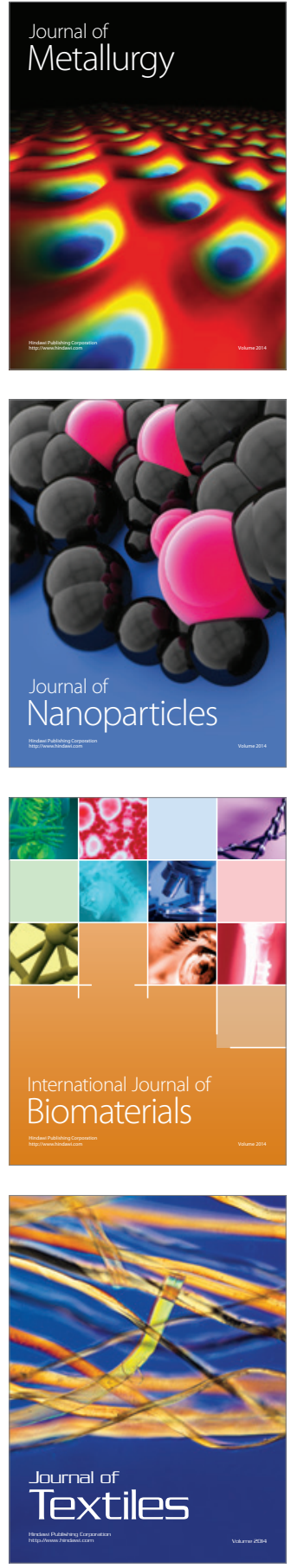